\section{Competencia Cultural en Salud: una necesidad urgente en trabajadores de la salud}

\section{Cultural Competence in health: an urgent need for health workers}

\section{Sr. Editor:}

La competencia cultural es entendida en el ámbito de la salud como la habilidad que deben poseer los trabajadores de la salud (TS) para incorporar una visión del contexto social y cultural de cada usuario, incrementando así la posibilidad de lograr una real comunicación con este último. Habitualmente, la necesidad de desarrollar la competencia cultural es entendida en la interacción que establecen los TS con la población de origen étnico o incluso en la interacción con inmigrantes internacionales, grupos en los cuales resulta más evidente la posible diferencia en el background cultural entre TS y usuario. Sin embargo, algunos autores $^{1-3}$ plantean que cada interacción en el ámbito de la salud debiera considerar una adaptación cultural de las estrategias, de tal forma que las intervenciones contemplen la individualidad y el contexto social de los usuarios, apostando de esta forma al éxito en la implementación de dichas intervenciones.

Considerando el perfil epidemiológico actual de nuestro país, en que predominan condiciones crónicas de salud, dos de los grandes desafíos que enfrentan los TS son la comunicación efectiva con los usuarios y la adherencia a las intervenciones sugeridas. Tomando como referencia el modelo de determinantes sociales de la salud ${ }^{4}$, es posible establecer que variables tales como género, edad, nivel educacional, situación laboral, nivel de ingresos, etnia o condición de migrante, sitúan a cada individuo en un entramado social complejo, el cual influye en sus decisiones de salud. Necesariamente estas variables debieran ser tomadas en cuenta por los TS para el diseño de estrategias que apunten al éxito y adherencia deseados.

En el ámbito de la atención primaria en salud esta habilidad de comunicarse efectivamente con el usuario, considerando su background cultural, pareciera tener especial relevancia, ya que es en este nivel donde debiera ocurrir el primer encuentro entre los usuarios y el sistema de salud, y generalmente es allí donde las personas controlan sus condiciones crónicas de salud. La Organización
Mundial de la Salud, en su documento de trabajo acerca de los lineamientos para Salud Primaria ${ }^{5}$, plantea que el cuidado de las personas debe centrarse en sus necesidades específicas, considerando la diversidad cultural de las comunidades.

Lamentablemente, en muchas ocasiones las intervenciones propuestas por los trabajadores de la salud parecieran obedecer a una "estandarización" de los cuidados, que se refleja, por ejemplo, en las indicaciones de tratamiento que recibe un paciente diabético o hipertenso que recibe un listado de indicaciones de salud que no necesariamente consideran sus condiciones de vida y el complejo entramado social en que se encuentra inmerso.

La incorporación de competencias culturales en las carreras de la salud es escasa en nuestro país, y actualmente no existe una herramienta que nos permita evaluar la competencia cultural que poseen nuestros TS. Pareciera necesario abordar esta temática desde la formación temprana para que de esta forma los TS adquieran herramientas efectivas para el logro de la competencia cultural.

\section{Margarita Bernales ${ }^{1,2, a}$, Víctor Pedrero ${ }^{2,3}$, Alexandra Obach ${ }^{2,4, a}$, Claudia Pérez ${ }^{5, c}$ ${ }^{1}$ Universidad de Auckland, Nueva Zelanda. ${ }^{2}$ Facultad de Medicina, Universidad del Desarrollo, Santiago de Chile. ${ }^{3}$ Pontificia Universidad Católica de Chile. ${ }^{4}$ Universidad de Barcelona. \\ ${ }^{5}$ Escuela de Enfermería, Facultad de Medicina Universidad del Desarrollo, Chile. ${ }^{a} \mathrm{PhD}$. \\ ${ }^{b}$ Enfermero, $M g .(C)$. 'Enfermera, Magíster en Psicología de la Salud.}

\section{Referencias}

1. Helman C. Doctor-Patient Interactions. Culture, Health, and Illness (Fifth Edition). Oxford: Oxford University Press; 2007. p. 121-55.

2. Kleinman A. Interpreting Illness Experience and Clinical Meanings: How I See Clinically Applied Anthropology Medical Anthropology Quarterly [Internet]. 1985; 16 (3): [69-71 pp.]. Available from: http://www.jstor.org/di scover $/ 10.2307 / 648599$ ? uid=3737784\&uid=2134\&uid= 2\&uid=70\&uid=4\&sid=21101105952031.

3. Kleinman A, Eisenberg L, Good B. Culture, illness, and care: clinical lessons from anthropologic and crosscultural research. Ann Intern Med [Internet]. 1978; 88 
(2): [251-8 pp.]. Available from: http://www.ncbi.nlm. nih.gov/pubmed/626456.

4. World Health Organization [WHO]. Closing the gap in a generation: Health equity through action on the social determinants of health 2008. Available from: http:// www.who.int/social_determinants/thecommission/ finalreport/en/index.html.

5. World Health Organization [WHO]. The World Health Report 2008-Primary Health Care: Now More Than Ever 2008 [cited 200906 July ]. Available from: http://www. who.int/whr/2008/whr08_en.pdf.
Carta generada en el Proyecto Fondecyt N 11130042

"Desarrollando Inteligencia en Salud Pública para inmigrantes en Chile: un estudio multi-métodos".

Direcciones electrónicas de los autores:

margaritabernales@udd.cl

vpedrero@udd.cl

aobach@udd.cl

claudiaperez@udd.cl 